\title{
Three-dimensional laser micro- and nanostructuring of acrylated polyethylene glycol materials and evaluation of their cytoxicity for tissue engineering applications
}

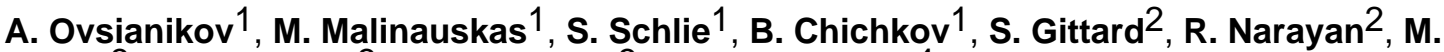 \\ Löbler $^{3}$, K. Sternberg ${ }^{3}$, K.-P. Schmitz ${ }^{3}$, and A. Haverich ${ }^{4}$ \\ ${ }^{1}$ Laser Zentrum Hannover, Hollerithallee 8, Hannover, Germany \\ ${ }^{2}$ Joint Department of Biomedical Engineering, University of North Carolina and North Carolina \\ State University, Chapel Hill, NC, USA \\ ${ }^{3}$ Institut für Biomedizinische Technik, Universität Rostock, Friedrich-Barnewitz-Str. 4, Rostock, \\ Germany \\ ${ }^{4}$ Medizinische Hochschule Hannover, Carl-Neuberg-Strasse 1, 30625 Hannover, Germany
}

\begin{abstract}
The natural cell environment is characterised by complex three-dimensional structures, which contain features at multiple length scales. Many in vitro studies of cell behaviour in three dimensions rely on the availability of artificial scaffolds with controlled three-dimensional topologies. In this paper, we demonstrate fabrication of three-dimensional scaffolds for tissue engineering out of poly(ethylene glycol) diacrylate (PEGda) materials by means of two-photon polymerization (2PP). This laser nanostructuring approach offers unique possibilities for rapid manufacturing of three-dimensional structures with arbitrary geometries. The spatial resolution dependence on the applied irradiation parameters is investigated for two PEGda formulations, which are characterized by molecular weights of 302 and 742 . We demonstrate that minimum feature sizes of $200 \mathrm{~nm}$ are obtained in both materials. In addition, an extensive study of the cytotoxicity of the material formulations with respect to photoinitiator type and photoinitiator concentration is undertaken. Aqueous extracts from photopolymerized PEGda samples indicate the presence of water-soluble molecules, which are toxic to fibroblasts. It is shown that sample aging in aqueous medium reduces the cytotoxicity of these extracts; this mechanism provides a route for biomedical applications of structures generated by $2 \mathrm{PP}$ microfabrication and photopolymerization technologies in general. Finally, a fully biocompatible combination of PEGda and a photoinitiator is identified. Fabrication of reproducible scaffold structures is very important for systematic investigation of cellular processes in three dimensions and for better understanding of in vitro tissue formation. The results of this work suggest that 2PP may be used to polymerize poly(ethylene glycol)-based materials into three-dimensional structures with welldefined geometries that mimic the physical and biological properties of native cell environments.
\end{abstract}

(c) 2010 Acta Materialia Inc. Published by Elsevier Ltd. All rights reserved.

Corresponding address: Dr. Aleksandr Ovsianikov, Laser Zentrum Hannover, Nanotechnology Department, Hollerithallee 8, Hannover, Germany, Phone: +49 (0) 5112788 233, Fax: +49 (0) 5112788 100, A.Ovsianikov@ gmail.com.

Publisher's Disclaimer: This is a PDF file of an unedited manuscript that has been accepted for publication. As a service to our customers we are providing this early version of the manuscript. The manuscript will undergo copyediting, typesetting, and review of the resulting proof before it is published in its final citable form. Please note that during the production process errors may be discovered which could affect the content, and all legal disclaimers that apply to the journal pertain. 


\section{Keywords}

polymer scaffolds; nanostructuring; two-photon polymerization; tissue engineering; cytotoxicity; poly(ethylene glycol) diacrylate

\section{Introduction}

Tissue engineering is a rapidly emerging field, which is dedicated to the development of novel technologies that address the challenges of tissue repair. One approach for generating artificial tissues involves placing cells within natural or synthetic scaffolds [1]. These scaffolds serve several purposes, which include (a) defining the overall geometry of the tissue substitute, (b) providing appropriate mechanical support for the seeded cells, and (c) introducing biological molecules to the seeded cells. Pore shape, size, distribution, and connectivity play important roles in determining scaffold performance. These factors influence the regeneration process and define the mechanical properties of the artificial tissue [2-4].

Using conventional approaches for scaffold fabrication (e.g., freeze-drying, phase separation, solvent casting, and electrospinning), it is now possible to control pore connectivity and pore size. Porosity levels of $\sim 90 \%$ can be realized, which provide sufficient pore connectivity for cell attachment and nutrient diffusion.

For control of cell migration and cellular interactions within the scaffold, technologies capable of producing three-dimensional structures in accordance with predefined designs are required. In recent years, rapid prototyping methods have been used for scaffold fabrication $[5,6]$. These methods include among others stereolithography, selective laser sintering, and fused deposition modeling. Fabrication of computer-aided design scaffolds with relatively large pores and spatial resolutions ranging from ten to hundreds of micrometers, depending on the method and used material, is possible with conventional rapid prototyping methods. So far, these rapid prototyping methods alone do not satisfy the requirements associated with mimicking of the natural cell environment. Moroni et al. recently described use of a combination of electrospinning and three-dimensional fiber deposition modeling for fabrication of scaffolds. The three-dimensional fiber deposited structure defines the geometrical and mechanical properties of the scaffold; the electrospun nanofibers within the channels serve as sieves for cell entrapment and provide topographical cues on the nanoscale [7].

Photopolymerization of materials provides an important alternative to conventional scaffold fabrication approaches. For example, aqueous solutions of poly(ethylene glycol) diacrylate (PEGda) materials can be used to produce photocrosslinkable hydrogels. Furthermore, it has been demonstrated that PEGda materials can be photopolymerized in direct contact with cells and tissues [8,9]. In addition, biodegradable formulations may be synthesized by chemical modification of PEGda materials [10]. Polymeric scaffold materials can be functionalized using proteins as well as other biological molecules in order to modulate cell adhesion [11-15]. Local biological molecule attachment in three dimensions within PEG hydrogels was also demonstrated by means of photopatterning [13-15]. This approach provides control over material-cell interactions by either preventing adhesion of cells or promoting adhesion of cells at specific locations. Papavasiliou et al. and Khademhosseini et al. demonstrated photopatterning of materials in order to obtain three-dimensional scaffolds; in these studies, conventional ultraviolet lithography and soft lithography methods were used to produce structures with features on the order of a few tens of micrometers [16,17]. It 
should be noted that both of these methods are inherently two-dimensional and require layer-by-layer processing in order to prepare three-dimensional scaffolds.

We have previously demonstrated the possibility of producing three-dimensional scaffolds from conventional photosensitive materials [18] and acrylated PEG materials [19] by means of two-photon polymerization (2PP). It is a rapid, single-step, and straightforward direct laser writing technology, which is based on nonlinear processing of photosensitive materials. The designs of 2PP-fabricated structures can be readily modified by altering computer-aided design files. The resolution of 2PP can be scaled by utilizing appropriate focusing optics and laser irradiation parameters. Structures with feature sizes ranging from less than $100 \mathrm{~nm}$ to hundreds of micrometers may be produced using this technique [20]. As such, 2PP allows one to produce scaffolds containing features at several length scales in a single step.

This work presents several studies involving photostructuring of photosensitive PEGda materials by means of two-photon polymerization. A minimum feature size of $200 \mathrm{~nm}$ is obtained for both tested materials. An important question for the application of any material for scaffold fabrication is its biocompatibility. In the case of photopolymerized materials, residual materials (e.g., photoinitiators or monomers), which are not consumed during the photopolymerization process, can impair cell viability. An extensive study to evaluate the cytotoxicity of material formulations with respect to photoinitiator type and photoinitiator concentration is conducted. A combination of PEGda material and photoinitiator that provides acceptable cell viability rates is identified.

\section{Materials and Methods}

\subsection{Two-photon polymerization (2PP) processing}

In this work, we utilize near-infrared Ti:sapphire femtosecond laser pulses (120 fs, $80 \mathrm{MHz}$, $780 \mathrm{~nm}$ ) for three-dimensional processing of materials. Similar to multiphoton microscopy, tightly focused femtosecond pulses interact with materials through the two-photon absorption process. If there is sufficient light intensity at the focus (i.e., the exposure dose exceeds the irradiation threshold for polymerization), a free-radical polymerization process is initiated (Fig. 1).

In the case of a liquid monomer (e.g., the PEGda formulations presented in this manuscript), the result of this light-material interaction is a localized conversion of liquid material to solid material. By moving the laser focus within the photosensitive resin, polymerization of material along the trace is obtained. This process allows the fabrication of a computergenerated three-dimensional structure with a defined geometry by direct laser "recording" into the volume of photopolymer [21].

For resolution studies of $2 \mathrm{PP}$ nanostructuring, the laser beam is focused into a droplet of photosensitive material using a $100 \times$ immersion oil microscope objective $(\mathrm{NA}=1.4)$ (Zeiss, Oberkochen, Germany). Single polymerized lines, which are supported by two parallel polymeric walls in order to keep the lines above the glass, are fabricated at different laser powers (Fig. 2b and Fig. 2c). After fabrication, unpolymerized material is washed away with distilled water. For the fabrication of scaffolds, an Epiplan 20× microscope objective $(\mathrm{NA}=0.4)$ (Zeiss, Oberkochen, Germany) is used.

\subsection{Materials}

In the present study, two different PEGda materials having molecular weights of 302 (SR259) (Sartomer, Paris, France) and 742 (SR610) (Sartomer, Paris, France) are used. Both materials are transparent to the applied laser radiation. In order to obtain a photopolymerizable material, a photoinitiator is added. The photoinitiator Michler's ketone 
(4,4' -bis(diethylamino)benzophenone) (Sigma-Aldrich, St. Louis, MO, USA) is used with the PEGda materials for 2PP structuring studies. For the cytocompatibility studies, two additional commercially available photoinitiators, Irgacure 369 and Irgacure 2959 (Ciba, Basel, Switzerland), are examined for comparison purposes. All photoinitiators presented in this manuscript are sensitive in the UV range. The photoinitiators are stirred directly into the poly(ethylene glycol) diacrylate material. All photoinitiator concentrations are determined by percent weight.

\subsection{Sample preparation for cytotoxicity studies and gravimetric analysis}

For cytotoxicity studies and gravimetric analysis studies, polymeric pellets having diameters of $6 \mathrm{~mm}$ and thicknesses of $1 \mathrm{~mm}$ are used. These samples are prepared from both material formulations by means of photopolymerization with ultraviolet light.

Material swelling is gravimetrically quantified. Freshly prepared pellets are weighed and immersed in water. After $24 \mathrm{~h}$, hydrated pellets are removed from the water, patted dry, and weighed again. It has been confirmed by our experiments that $24 \mathrm{~h}$ is a sufficient immersion time to reach equilibrium water sorption; longer immersion times do not contribute to further swelling. After drying, the hydrated pellets are placed in a convection oven for $2 \mathrm{~h}$ at $60^{\circ} \mathrm{C}$; these dried materials are shown to exhibit their original weight. The amount of water absorbed by the material is characterised by a swelling ratio $\left(100 \times\left(\mathrm{W}_{\text {swollen }}-\mathrm{W}_{\mathrm{dry}}\right) / \mathrm{W}_{\mathrm{dry}}\right.$ [\%]).

\subsection{Analysis of material cytotoxicity by the extract test}

Elution of cytotoxic substances from the material into the surrounding aqueous medium is assayed in the extract test. Sterile material pellets are soaked for $24 \mathrm{~h}$ at $37^{\circ} \mathrm{C}$ in cell culture medium (DMEM) lacking fetal calf serum (FCS) under continuous agitation. A ratio of test material surface area to cell culture medium of $6 \mathrm{~cm}^{2}$ material surface area to $1 \mathrm{~mL}$ of culture medium is used according to ISO10993. The resulting extract is supplemented with $10 \%$ FCS and is serially diluted with cell culture medium. All extracts and extract dilutions are tested for cytotoxicity in quadruplicate using ATCC L929 mouse fibroblasts (DSMZ, Braunschweig, Germany). L929 mouse fibroblasts are seeded at a density of 2000 cells/well in a 96 well microtiter plate; the seeded cells are incubated for $24 \mathrm{~h}$ at $37^{\circ} \mathrm{C}, 5 \% \mathrm{CO}_{2}$ at $95 \%$ relative humidity. The cell culture medium is subsequently replaced with the material extract solution and the cells are incubated for an additional $48 \mathrm{~h}$. This extract solution will hereunto be referred to as the extract from freshly prepared material. The extract solution is then removed and the cells are incubated for $2 \mathrm{~h}$ in a solution of $10 \%$ CellQuanti-Blue (BioAssay Systems, Hayward, CA, USA) in cell culture medium. Then fluorescence is determined in a Fluostar Optima plate reader (BMG Labtech, Offenburg, Germany) at 544 $\mathrm{nm}$ excitation and $590 \mathrm{~nm}$ emission wavelengths [22]. The fluorescence intensity data is taken as a measure of cell viability.

Following extraction, material pellets are briefly rinsed with distilled water and then aged for 6 days at $37^{\circ} \mathrm{C}$ under agitation in sterile distilled water, which is changed on a daily basis. The material pellets are extracted a second time in cell culture medium as described above; the extracts are then tested for their cytotoxic potential. The material pellets used in the second extraction test will be referred to as "aged" samples for the remainder of this manuscript.

Extracts from glass, Teflon ${ }^{\circledR}$, and cell culture medium without any material exposure served as negative controls. As a positive control, cells are incubated with cytotoxic tetraethylthiuram disulfide (TETD) (concentration $=10^{-5} \mathrm{M}$ ) in cell culture medium. 


\subsection{Cell viability and adhesion in direct contact with test material}

For the direct contact test, freshly prepared or aged (soaked in culture medium for $24 \mathrm{~h}$ ) material discs (diameter $=6 \mathrm{~mm}$ ) are sterilized by autoclaving. The discs are then placed into the wells of a 96-well microtiter plate. 4000 L929 mouse fibroblasts are seeded onto the discs in $200 \mu 1$ of cell culture medium per well. After $48 \mathrm{~h}$ of cultivation, a cell viability test is performed with the CellQuanti-Blue viability assay (BioAssay Systems, Hayward, CA, USA). The culture medium in each well is replaced with $200 \mu \mathrm{l}$ of $10 \%$ cell Quanti-Blue reagent in cell culture medium; microtiter plates are subsequently processed as described in section 2.4. A polystyrene microtiter plate and a glass cover slip serve as negative controls. As positive control, a cytotoxic ZDEC membrane (Hatano Research Institute, Kanagawa, Japan) and TETD (concentration $=10^{-5} \mathrm{M}$ ) in culture medium is used.

Cell adhesion is further evaluated by a live/dead assay (Invitrogen, Darmstadt, Germany) and by confocal laser scanning microscopy. Membrane permeable calcein-AM is converted intracellularly into calcein, which is not membrane permeable; this mechanism is used to stain the cytoplasms of living cells. At the same time, DNA located in the nuclei of dead cells is stained with membrane impermeable ethidium-dimer, resulting in a red fluorescence signal. For the rapid identification of all the cell nuclei, Hoechst 33342 (Invitrogen, Mountain View, CA, USA) is used to stain DNA in both viable and dead cells, resulting in a blue fluorescence signal. Fluorescently labelled cells are visualized with a FV1000 confocal laser scanning microscope (Olympus, Hamburg, Germany).

\section{Results}

\subsection{Two-photon polymerization (2PP) microfabrication}

For resolution studies of the 2PP process, a laser beam is focused into a droplet of photosensitive material using a 100 $\times$ immersion oil microscope objective $(\mathrm{NA}=1.4)$ (Zeiss, Oberkochen, Germany). In order to determine the minimum resolution for each material, structures like those shown in Fig. $2 \mathrm{~b}$ are produced. Two parallel walls support a set of parallel lines that are sequentially photopolymerized by means of a single scan; various average laser powers are utilized for the single scans that span the two supports. The scanning speed is maintained at a constant value of $200 \mu \mathrm{m} / \mathrm{s}$ throughout the experiment. Lower average powers result in smaller illumination doses and smaller line dimensions. Due to the nature of the $2 \mathrm{PP}$ technique, these lines demonstrate elliptical cross-sections, which are elongated along the laser beam propagation direction. Line heights and line widths are plotted against average laser power (Fig. 2a). For the SR259 material, the minimum lateral resolution $(200 \mathrm{~nm})$ is obtained at an average laser power of $27 \mathrm{~mW}$. A similar resolution is obtained for the SR610 material; a lower applied average power $(22 \mathrm{~mW})$ is utilized in this case. Lines produced slightly below these illumination conditions did not survive the development procedure. In general, cross-sections of single lines produced by means of $2 \mathrm{PP}$ with the focusing optics used here should exhibit aspect ratios larger than 2.5. It is observed that line aspect ratios are generally smaller for SR610 materials. Since the applied focusing conditions are not varied in these experiments, this difference can be attributed to the higher shrinkage of SR610. Residual strain in the structure results in a rather symmetrical line profile as well as in a reduced aspect ratio. Our previous studies with another material also confirm that shrinkage in the microstructures tends to be anisotropic [23].

At the upper end of the applied average power range (close to $140 \mathrm{~mW}$ ), 2PP of SR610 material commonly results in patterns such as the one shown in Fig. 2c. The membrane-like structure observed in the upper part of the image does not result from direct laser irradiation since only lines are scanned. We attribute these patterns to diffusion-driven polymerization. Free radicals, which are produced during processing of the walls and the separate lines 
between them, disperse and cause polymerization outside of the laser-exposed region. Most of these membranes are not complete and some holes are observed; these features indicate that the membrane-like structures possess poor mechanical stability.

Fig. 3 shows a prototype of a three-dimensional scaffold structure fabricated by 2PP using SR610 material. It is composed of $50 \mu \mathrm{m}$ tall hollow cylinders (radius $=25 \mu \mathrm{m}$ ) in a square arrangement within each layer. Every second layer is shifted by the length of the cylinder diameter along the diagonal. Most of the cylinders in each layer partially overlap with four different cylinders of the next layer (Fig. 3c). The presented structure is characterized by interpenetrating pores with different sizes. Individual cylinders may serve to accommodate cells; the spaces in between the cylinders may serve to provide routes for fluid transport. Since the structure exhibits relatively large dimensions, a 20X microscope objective (Epiplan, NA=0.4) (Zeiss, Oberkochen, Germany) is used to focus laser pulses into the material during $2 \mathrm{PP}$ processing.

\subsection{Material swelling ratio}

Gravimetric analysis of material swelling shows that SR610 samples swell up to $60 \%$ of their initial weight after immersion for $24 \mathrm{~h}$ in PBS. The hydrated pellets are placed in a convection oven for $2 \mathrm{~h}$ at $60^{\circ} \mathrm{C}$; after drying they retain their original weight. On the other hand, no gravimetrically measurable swelling is observed for SR259.

\subsection{Material cytotoxicity with respect to photoinitiator type and concentration}

Cytotoxicity testing of photopolymer extracts is performed in order to analyze the effects of material composition on cell viability. The extracts are obtained from photopolymerized samples, which contain different photoinitiators and photoinitiator concentrations. Formulations containing large amounts of photoinitiator result in higher levels of cytotoxicity. Furthermore, aging of samples in distilled water significantly reduces toxicity levels.

Figure 4 shows the results obtained with SR610 samples that are photopolymerized in the presence of 2\% wt Michler's ketone photoinitiator. It is observed that the viability of L929 fibroblasts is suppressed at an extract concentration as low as $3.125 \%$. Aging of the samples in distilled water for six additional days reduces the amount of water-soluble toxic components such that cytotoxicity occurs at extract concentrations above $12.5 \%$ (Fig. 4). The inhibitory extract concentration at which the cell viability is reduced by $50 \%\left(\mathrm{IC}_{50}\right)$ is $21 \%$ for the aged samples.

Samples of SR610 materials with $0.5 \%, 1 \%, 2 \%$, and 3\% wt Irgacure 369 photoinitiator are also prepared. Extracts prepared from SR610 containing Irgacure 369 show similar cytotoxic effects for photoinitiator concentrations of $0.5 \%, 1 \%$, and $2 \% \mathrm{wt}$; a photoinitiator concentration of $3 \% \mathrm{wt}$ is clearly more toxic (Fig. 5a). The inhibitory extract concentrations at which the cell viability is reduced by $50 \%\left(\mathrm{IC}_{50}\right)$ are $13 \%, 37 \%, 56 \%$ and $45 \%$ for the formulations containing 3\%, 2\%, $1 \%$ and $0.5 \%$ wt Irgacure 369 photoinitiator, respectively. Cell viability in the undiluted extract from freshly prepared SR610 containing Irgacure 369 is below $10 \%$ for all photoinitiator concentrations.

In contrast, extracts obtained after aging the samples for six days in distilled water show substantially reduced cytotoxic potential values (Fig. $5 \mathrm{~b}$ ). The $\mathrm{IC}_{50}$ values for extract concentration are $63 \%, 83 \%$, and $>100 \%$ for formulations containing $3 \%, 2 \%$, and $1 \%$ or $0.5 \%$ wt Irgacure 369 photoinitiator, respectively. Extracts from freshly prepared SR610 and aged SR610 containing Irgacure 369 may be considered cytotoxic. 
Figure 6a presents results of tests performed with SR610 formulations containing 2\% Irgacure 2959 photoinitiator and $0.5 \%$ Irgacure 2959 photoinitiator. Again, it is observed that higher concentrations of the photoinitiator in the freshly prepared samples are associated with higher cytotoxicity levels. The $\mathrm{IC}_{50}$ value for $2 \%$ wt Irgacure 2959 is $36 \%$ extract concentration and the $\mathrm{IC}_{50}$ value for $0.5 \% \mathrm{wt}$ Irgacure 2959 is $45 \%$ extract concentration. However, both samples may be regarded to as cytotoxic. Aging of the samples in distilled water for an additional 6 days reduces the amount of the water-soluble toxic components to levels at which no cytotoxic effect is observed (Fig. 6a). Therefore, the photoinitiator and possibly other toxic components can be washed out of 2PP-fabricated structures, providing a fully biocompatible PEGda material.

\subsection{Cell viability and adhesion in direct contact}

The extracts of aged pellets prepared with the photoinitiator Irgacure 2959 exhibit low cytotoxicity; material discs of this material (diameter $=6 \mathrm{~mm}$ ) are seeded with $\mathrm{L} 929$ mouse fibroblasts and examined for cell viability. It is observed that freshly prepared samples again strongly impair cell viability; on the other hand, aged samples are only moderately cytotoxic (Fig. 6b). In the extract test (Fig. 6a) and the direct contact test (Fig. 6b), cell viability inversely correlates with the amount of photoinitiator. Confocal microscopy images (Fig. 7) show that cells grown on aged SR610 samples exhibit rounded morphologies; this cell shape is an indication of poor cell attachment to the substrate. Confocal laser scanning microscopy images show both viable and dead cells on the SR610 samples. A quantitative evaluation was not performed due to an inhomogeneous distribution of cells on the samples.

\section{Discussion}

Artificial tissues must provide a cell environment similar to that of natural tissues. Scaffold materials used in artificial issues would benefit from well-controlled topologies, which may be used to alter cellular behavior in a desired manner. Recent studies have indicated that micro- and nanopatterned substrates may be used to create direct cell-substrate interactions and stem cell fate [24-26]. In this work, we present a method known as 2PP for fabrication of computer-aided design fabricated scaffolds, which exhibit great precision and reproducibility. A minimum lateral resolution of $\sim 200 \mathrm{~nm}$ is obtained for the two PEGda formulations. Due to the high resolution of the $2 \mathrm{PP}$ technique, it is possible to produce scaffolds with features at several length scales in a single fabrication step. Further control over cell-scaffold interaction may be introduced via alterations to the surface topographies of the 2PP-fabricated scaffolds.

Membrane-like patterns, which result from diffusion-driven polymerization, are observed on structures fabricated from SR610 material. If these features can be obtained in a controllable manner, membrane-like patterns might find applications in isolating cells within threedimensional scaffolds. Recent reports have described isolation of embryonic pancreatic precursor cells in PEG-based hydrogels for controlling cell differentiation [27,28]. Membrane-like features produced by 2PP may also be used for isolating various types of cells within a given scaffold.

The water sorption data for $2 \mathrm{PP}$-fabricated PEGda structures provides two interesting considerations. First, water sorption leads to an increase in volume of 2PP-fabricated SR610 structures. This process will lead to deviations of structures from their original geometries. Second, water sorption will result in enhanced porosity, which will increase fluid transport within the scaffold. This result also suggests that clinically-relevant biological materials (e.g., growth factors or antimicrobial agents) can be delivered to tissues using appropriately loaded PEGda scaffolds. Both growth factors and antimicrobial agents have previously been encapsulated within PEGda materials [29-31]. 
Photopolymerization of the SR259 material results in rigid structures, which do not absorb a significant amount of water. This rigid material is useful for applications in which swelling is undesirable (e.g., biomedical devices and microscale prostheses). A combination of various PEGda materials with different molecular weights may possess intermediate mechanical properties as well as intermediate swelling properties.

Cytotoxicity of SR610 with three different commercially available photoinitiators, including Michler's ketone, Irgacure 369 and Irgacure 2959, was investigated. A comparison of IC $_{50}$ data indicates that Michler's ketone exhibits the highest cytotoxicity (Fig. 4). It is shown that the toxicity levels associated with the presence of residual material after photopolymerization can be significantly reduced by aging the samples in water. The combination of SR610 and Irgacure 2959 is shown to exhibit the low cytotoxic potential (Fig. 6). The moderate cytotoxicity of aged samples in the direct contact test is attributed to residual toxicity and local presence of toxic photoinitiator. Reduced cell viability may also result from the surface characteristics of the poly (ethylene glycol) material. Poly (ethylene glycol) exhibits inert, antifouling behavior due to the fact that uncharged hydrophilic groups in the material are exposed in aqueous solutions; these groups form hydrogen bonds with water molecules [32]. Polyethylene glycol diacrylate-based materials exhibit nonspecific resistance to cell and protein adhesion due to these interactions [33-35]. Adding an adhesive ligand to the formulation may improve cell attachment and cell viability. Since Irgacure 2959 is the only water-soluble photoinitiator that was examined, we conclude that the toxic effects in the freshly prepared samples are associated with photoinitiator molecules that are not consumed during the photopolymerization process. This finding is also supported by the fact that toxic effects are increased in materials containing higher initial photoinitiator concentrations for all examined photoinitiator types. We anticipate that toxicity may be minimized by using appropriate solvents as aging media as well as photoinitiators that may easily be removed through placement in an appropriate solvent. Studies of cell viability and cell adhesion involving photopolymerized PEGda are in good correlation with cytotoxicity experiments; cell viability is substantially improved for aged samples. Sample aging and postprocessing procedures may facilitate biological or medical use of structures fabricated using PEGda by means of two-photon polymerization.

\section{Conclusions}

In summary, microscale and nanoscale structuring of poly(ethylene glycol) diacrylate materials by two-photon polymerization has been examined for the first time to the best of our knowledge. This work shows that structures fabricated using poly(ethylene glycol) diacrylate material with relatively high molecular weights are more affected by shrinkage and have less well-defined geometries upon sorption of water. Swelling of two-photon polymerization-fabricated three-dimensional structures in aqueous solutions may enable the development of scaffolds with useful biological molecule encapsulation and release properties. In the same manner, possible residual materials (e.g., photoinitiator or monomer molecules) that are not consumed during the photopolymerization reaction may diffuse out of the polymer and impair cell viability. In this paper, it has been shown that aging of the photopolymerized materials significantly reduces material toxicity to levels at which these materials are no longer cytotoxic. These findings demonstrate that poly(ethylene glycol) diacrylate materials processed by means of two-photon polymerization can be used as biocompatible three-dimensional scaffolds. The use of two-photon polymerization for the nanostructuring of poly(ethylene glycol) materials is a combination of a flexible manufacturing technology and a promising material platform, which may be used to fabricate structures for a wide variety of medical applications. Due to the small feature sizes that can be obtained using two-photon polymerization, three-dimensional scaffolds containing features at several length scales can be produced in a single fabrication step. The 
post-processing protocol described in this manuscript provides the basis for creating biocompatible three-dimensional scaffolds from photopolymerized poly(ethylene glycol).

\section{Acknowledgments}

The presented work was supported by the DFG Excellence Cluster "Rebirth" and the SFB Transregio 37, "Mikround Nanosysteme in der Medizin - Rekonstruktion biologischer Funktionen".

\section{References}

1. Ma, PX.; Elisseeff, JH. Scaffolding in tissue engineering. Boca Raton, FL (USA): CRC Press; 2005.

2. Hutmacher DW. Scaffold design and fabrication technologies for engineering tissues state of the art and future perspectives. Journal of Biomaterials Science, Polymer Edition 2001;12:107-124. [PubMed: 11334185]

3. Harley BAC, Kim HD, Zaman MH, Yannas IV, Lauffenburger DA, Gibson LJ. Microarchitecture of three-dimensional scaffolds influences cell migration behavior via junction interactions. Biophysical Journal 2008;95(8):4013-4024. [PubMed: 18621811]

4. Liu DM. Control of pore geometry on influencing the mechanical property of porous hydroxyapatite bioceramic. Journal of Materials Science Letters 1996;15(5):419-421.

5. Hutmacher DW, Sittinger M, Risbud MV. Scaffold-based tissue engineering: rationale for computer-aided design and solid free-form fabrication systems. Trends in Biotechnology 2004;22:354-362. [PubMed: 15245908]

6. Yeong WY, Chua CK, Leong KF, Chandrasekaran M. Rapid prototyping in tissue engineering: challenges and potential. Trends in Biotechnology 2004;22:643-652. [PubMed: 15542155]

7. Moroni L, Schotel R, Hamann D, de Wijn JR, van Blitterswijk CA. 3D Fiber-deposited electrospun integrated scaffolds enhance cartilage tissue formation. Advanced Functional Materials 2008;18:5360.

8. Liu VA, Bhatia SN. Three-dimensional photopatterning of hydrogels containing living cells. Biomedical Microdevices 2002;4(4):257-266.

9. Koh WG, Revzin A, Pishko MV. Poly(ethylene glycol) hydrogel microstructures encapsulating living cells. Langmuir 2002;18(7):2459-2462. [PubMed: 12088033]

10. Mann BK, Gobin AS, Tsai AT, Schmedlen RH, West JL. Smooth muscle cell growth in photopolymerized hydrogels with cell adhesive and proteolytically degradable domains: synthetic ECM analogs for tissue engineering. Biomaterials 2001;22(22):3045-3051. [PubMed: 11575479]

11. Vande Vondele S, Voros J, Humbell JA. RGD-grafted poly-l-lysine-graft-(polyethylene glycol) copolymers block non-specific protein adsorption while promoting cell adhesion. Biotechnology and Bioengineering 2003;82(7):784-790. [PubMed: 12701144]

12. Huang NP, Voros J, De Paul SM, Textor M, Spencer ND. Biotin-derivatized poly(l-lysine)-gpoly(ethylene glycol): A novel polymeric interface for bioaffinity sensing. Langmuir 2002;18(1): 220-230.

13. Hahn M, Miller J, West J. Three-dimensional biochemical and biomechanical patterning of hydrogels for guiding cell behavior. Advanced Materials 2006;18(20):2679-2684.

14. Lee S-H, Moon JJ, West JL. Three-dimensional micropatterning of bioactive hydrogels via twophoton laser scanning photolithography for guided 3D cell migration. Biomaterials 2008;29(20): 2962-2968. [PubMed: 18433863]

15. Hoffmann JC, West JL. Three-dimensional photolithographic patterning of multiple bioactive ligands in poly(ethylene glycol) hydrogels. Soft Matter 2010;6:5056-5063.

16. Papavasiliou G, Songprawat P, Perez-Luna V, Hammes E, Morris M, Chiu Y-C, et al. Threedimensional patterning of poly(ethylene glycol) hydrogels through surface-initiated photopolymerization. Tissue Engineering Part C: Methods 2008;14(2):129-140. [PubMed: 18471086]

17. Khademhosseini A, Yeh J, Jon S, Eng G, Suh KY, Burdick JA, Langer R. Molded polyethylene glycol microstructures for capturing cells within microfluidic channels. Lab on a Chip 2004;4(5): 425-430. [PubMed: 15472725] 
18. Ovsianikov A, Schile S, Ngezahayo A, Haverich A, Chichkov BN. Two-photon polymerization technique for microfabrication of CAD-designed 3D scaffolds from commercially available photosensitive materials. Journal of Tissue Engineering and Regenerative Medicine 2007;1(6): 443-449. [PubMed: 18265416]

19. Ovsianikov A, Ostendorf A, Chichkov BN. Three-dimensional photofabrication with femtosecond lasers for applications in photonics and biomedicine. Applied Surface Science 2007;253(15): 6599-6602.

20. Ovsianikov, A.; Passinger, S.; Houbertz, R.; Chichkov, B. Three dimensional material processing with femtosecond lasers. In: Phipps, C., editor. Laser Ablation and its Applications, Springer Series in Optical Science. 2007. p. 121-157.

21. Maruo S, Nakamura O, Kawata S. Three-dimensional microfabrication with two-photon-absorbed photopolymerization. Optics Letters 1997;22(2):132-134. [PubMed: 18183126]

22. Nociari MM, Shalev A, Benias P, Russo C. A novel one-step, highly sensitive fluorometric assay to evaluate cell-mediated cytotoxicity. Journal of Immunological Methods 1998;213(2):157-167. [PubMed: 9692848]

23. Ovsianikov A, Shizhou X, Farsari M, Vamvakaki M, Fotakis C, Chichkov BN. Shrinkage of microstructures produced by two-photon polymerization of Zr-based hybrid photosensitive materials. Optics Express 2009;17(4):2143-2148. [PubMed: 19219118]

24. Lim JY, Donahue HJ. Cell sensing and response to micro- and nanostructured surfaces produced by chemical and topographic patterning. Tissue Engineering 2007;13(8):1879. [PubMed: 17583997]

25. Flemming RG, Murphy CJ, Abrams GA, Goodman SL, Nealey PF. Effects of synthetic micro- and nano-structured surfaces on cell behavior. Biomaterials 1999;20(6):573-588. [PubMed: 10213360]

26. Dalby MJ, Gadegaard N, Tare R, Andar A, Riehle MO, Herzyk P, et al. The control of human mesenchymal cell differentiation using nanoscale symmetry and disorder. Nature Materials 2007;6(12):997-1003.

27. Mason MN, Mahoney MJ. Selective $\beta$-cell differentiation of dissociated embryonic pancreatic precursor cells cultured in synthetic polyethylene glycol hydrogels. Tissue Engineering Part A 2008;15(6):1343-1352. [PubMed: 19072086]

28. Ford MC, Bertram JP, Hynes SR, Michaud M, Li Q, Young M, et al. A macroporous hydrogel for the coculture of neural progenitor and endothelial cells to form functional vascular networks in vivo. Proceedings of the National Academy of Sciences of the United States of America 2006;103(8):2512-2517. [PubMed: 16473951]

29. Cai S, Liu Y, Shu ZH, Prestwich GD. Injectable glycosaminoglycan hydrogels for controlled release of human basic fibroblast growth factor. Biomaterials 2005;26(30):6054-6067. [PubMed: 15958243]

30. Gittard SD, Ovsianikov A, Akar H, Chichkov B, Monteiro-Riviere NA, Stafslien S, et al. Two photon polymerization-micromolding of polyethylene glycol-gentamicin sulfate microneedles. Advanced Engineering Materials 2010;12(4):B77-B82. [PubMed: 21037972]

31. Adiga SP, Curtiss LA, Elam JW, Pellin MJ, Shih CC, Shih CM, et al. Nanoporous materials for biomedical devices. JOM Journal of the Minerals, Metals, and Materials Society 2008;60(3):2632.

32. Gombotz WR, Guanghui W, Horbett TA, Hoffman AS. Protein adsorption to poly(ethylene oxide) surfaces. Journal of Biomedical Materials Research 1991;25:1547-1562. [PubMed: 1839026]

33. DeLong SA, Moon JJ, West JL. Covalently immobilized gradients of bFGF on hydrogel scaffolds for directed cell migration. Biomaterials 2005;26:3227-3234. [PubMed: 15603817]

34. Moon JJ, Hahn MS, Kim I, Nsiah BA, West JL. Micropatterning of poly(ethylene glycol) diacrylate hydrogels with biomolecules to regulate and guide endothelial morphogenesis. Tissue Engineering Part A 2009;15(3):579-585. [PubMed: 18803481]

35. Nagaoka S, Nakao A. Clinical application of antithrombogenic hydrogel with long poly (ethylene oxide) chains. Biomaterials 1990;11:119-121. [PubMed: 2317533] 
Near-IR light

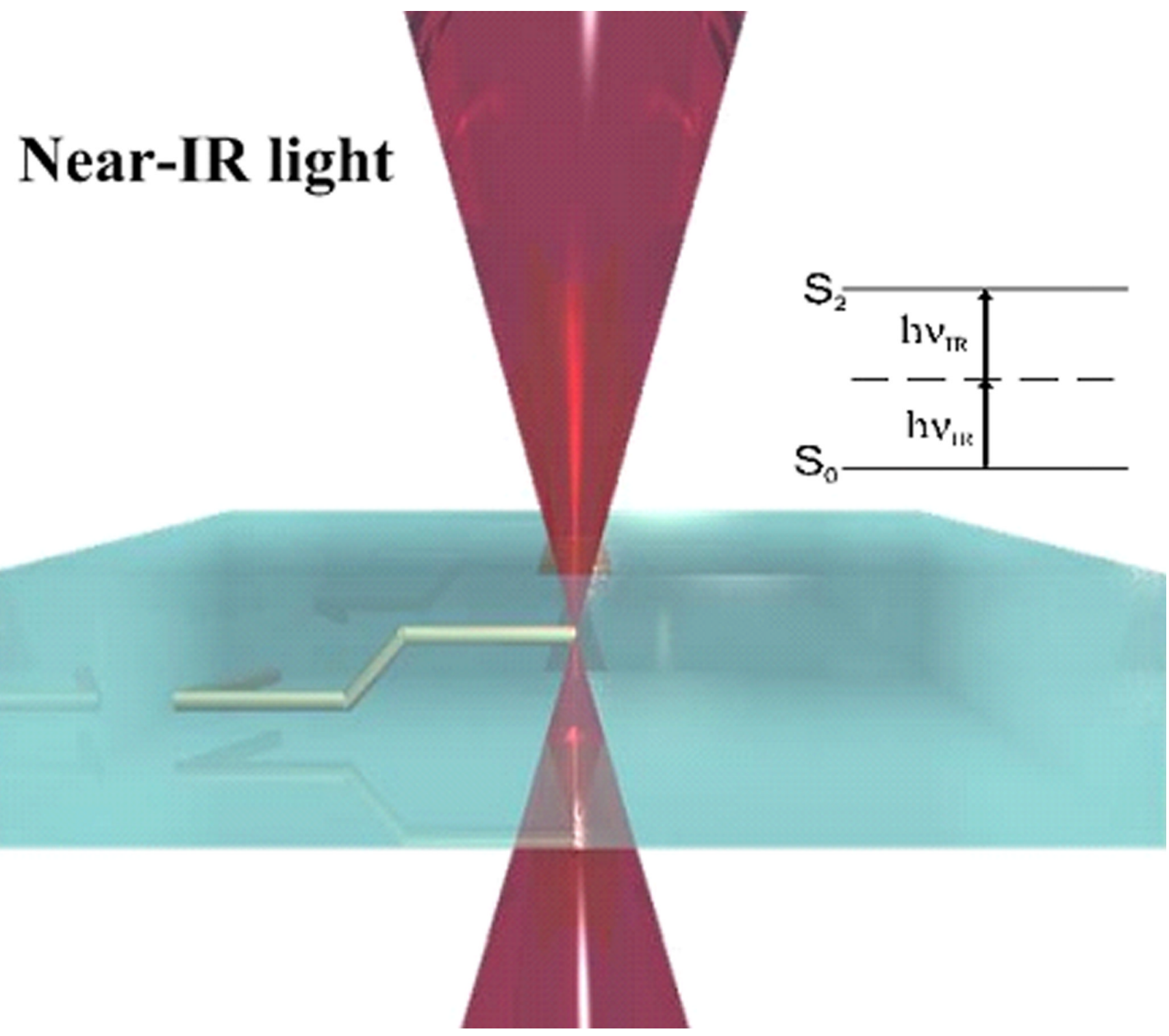

Figure 1.

Schematic illustration of two-photon polymerization process: polymerization is initiated by two-photon absorption in a high intensity region that is limited to the focal volume of the laser beam. By moving the laser focus within the material, three-dimensional patterns may be generated in a single step. 

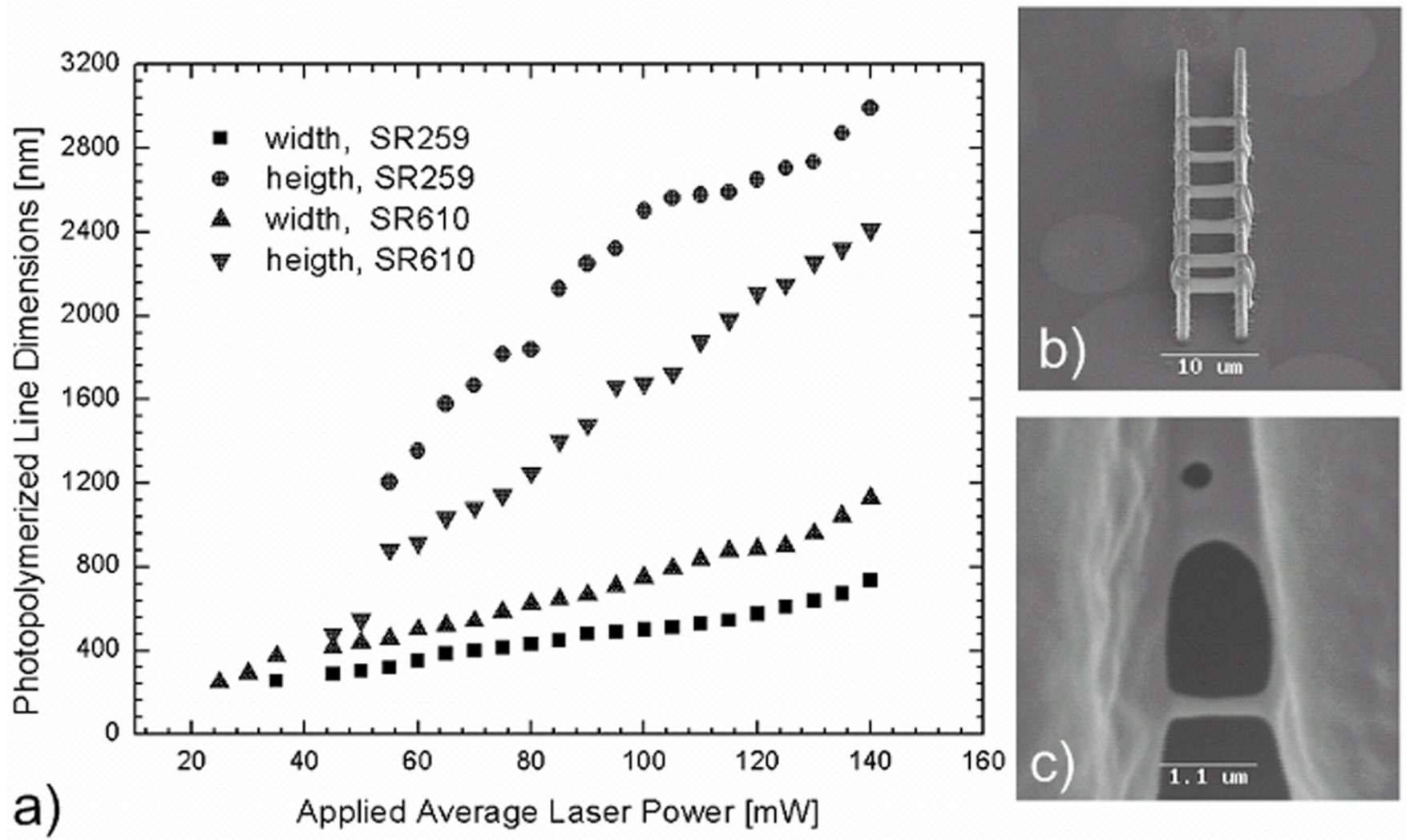

Figure 2.

a) Line width versus applied average laser power and height versus applied average laser power for two polyethylene glycol diacrylate formulations. b) An SEM image of a representative structure used for resolution studies. c) An SEM image of diffusion-driven patterns obtained with SR610 material. 

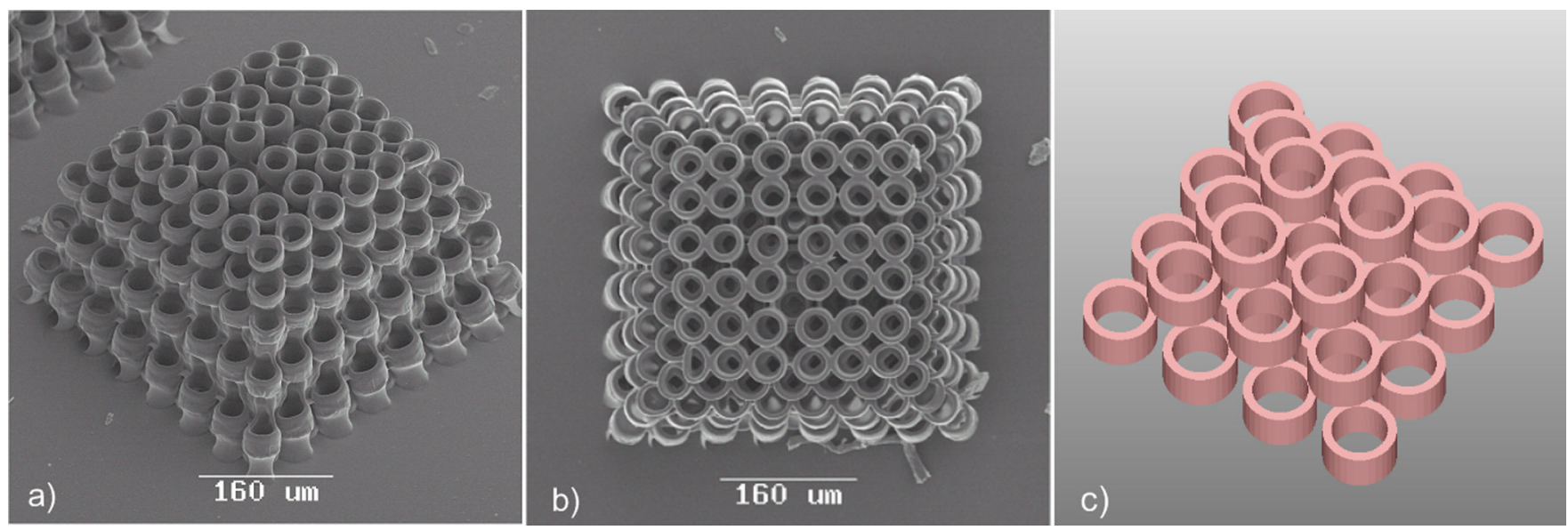

Figure 3.

Three-dimensional scaffold fabricated by two-photon polymerization of SR610 material: a) perspective view SEM image of produced structure; $b$ ) top view SEM image of produced structure; c) schematic illustration of cylinder arrangement. 


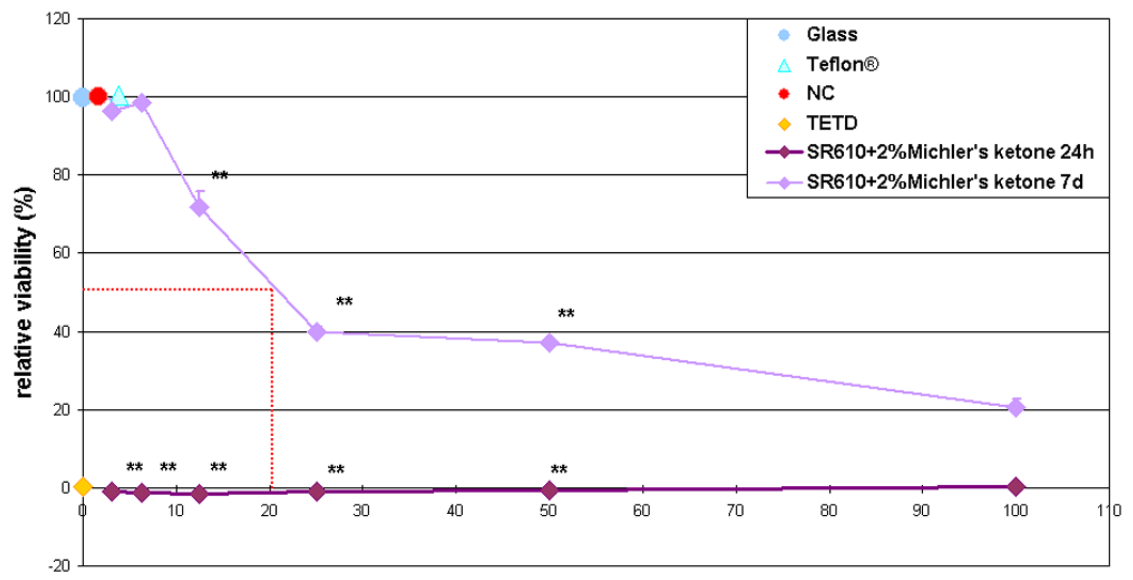

Sample extract (\%)

Figure 4.

Relative viability of L929 fibroblasts under the influence of extract from freshly prepared (24 h) and aged (7 d) SR610 samples, which were photopolymerized in the presence of 2\% Michler's ketone photoinitiator. Aged samples exhibit substantially reduced cytotoxic potential. 

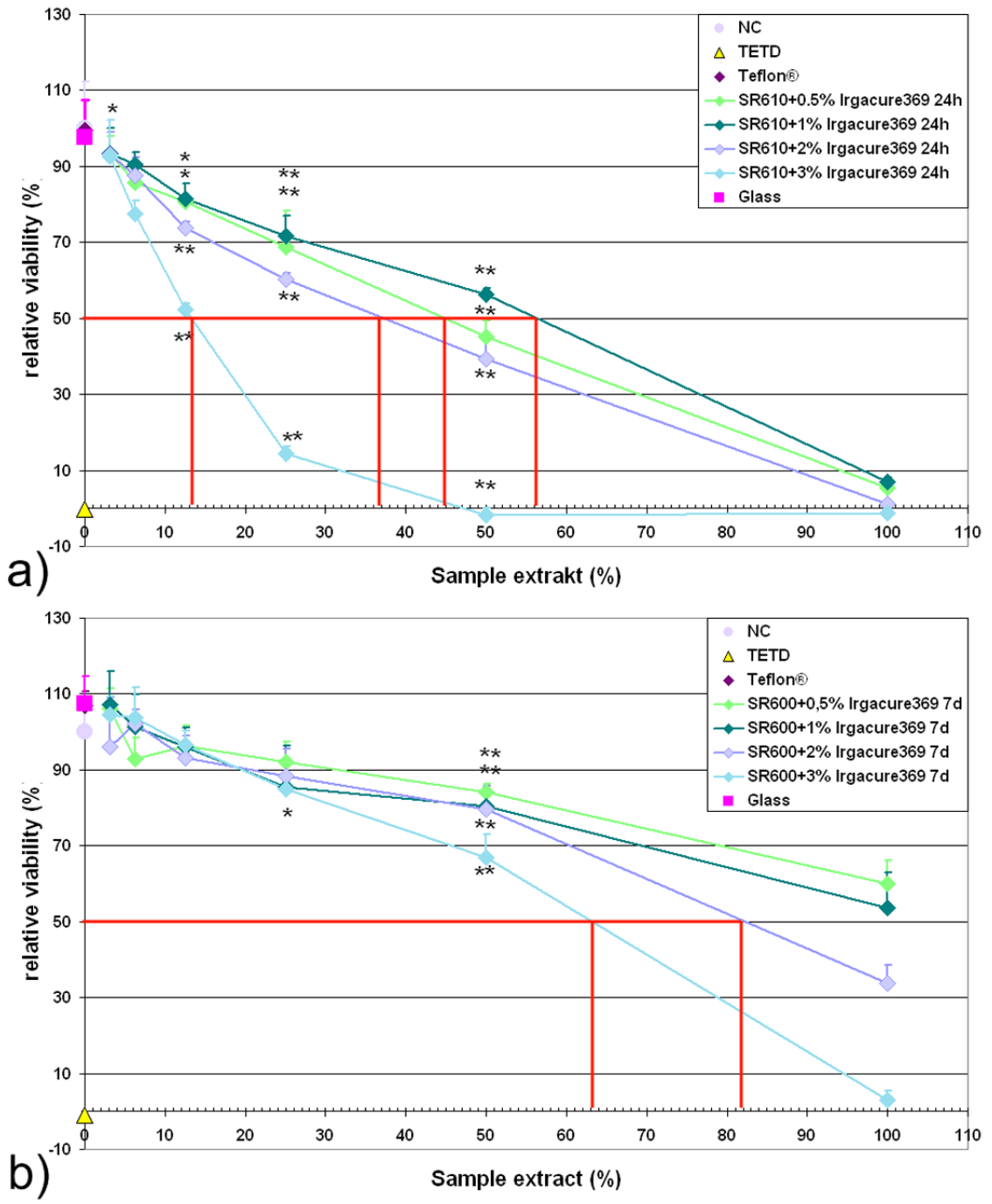

Figure 5.

Relative viability of L929 fibroblasts in response to extract from (a) freshly prepared (24 h) and (b) aged (7 d) SR610 samples, which contain photoinitiator Irgacure 369 in concentrations between $0.5 \%$ and $3 \%$. Relative cell viability correlates inversely with the amount of photoinitiator; aged samples exhibit substantially reduced cytotoxic potential. 

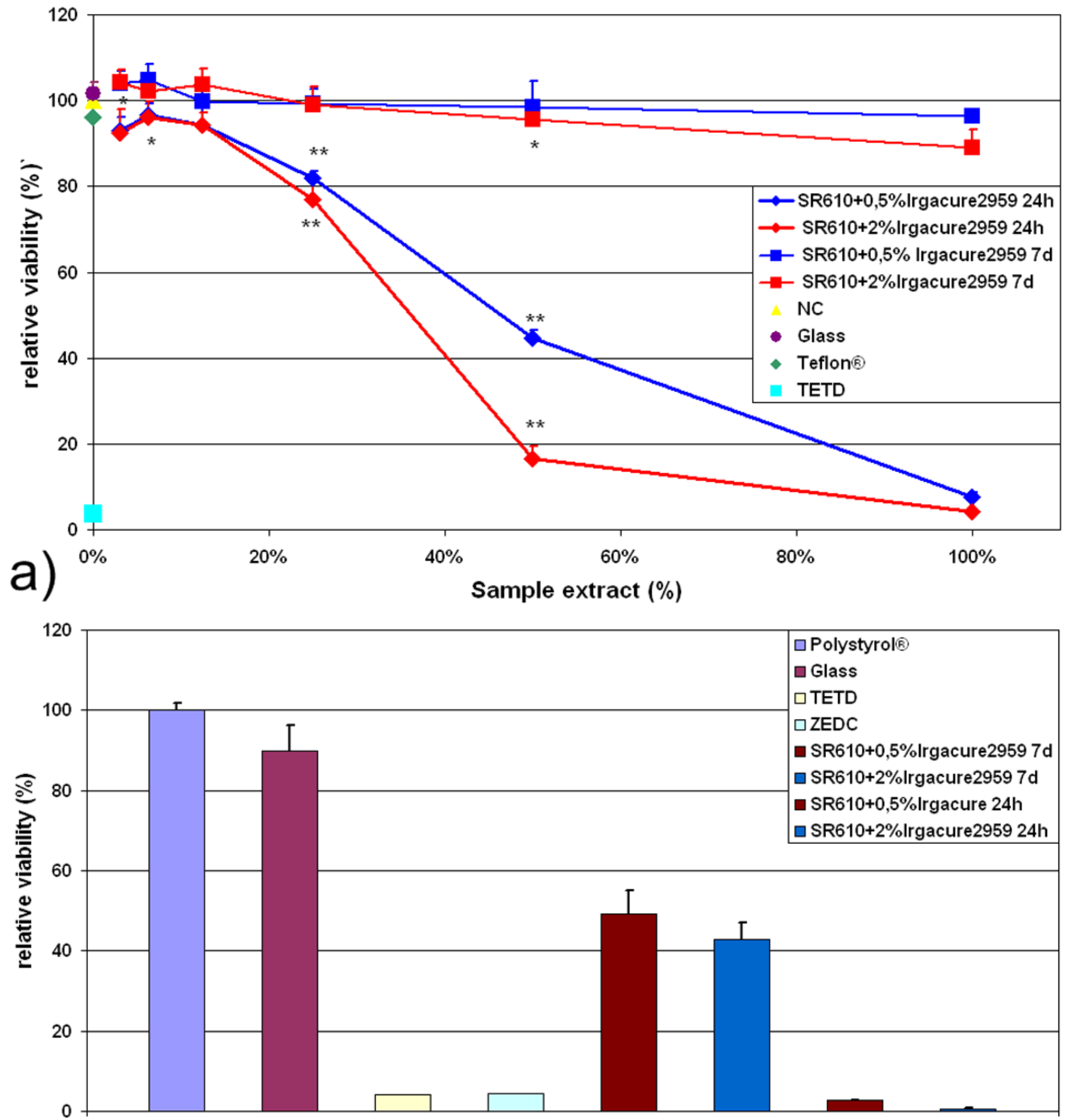

b)

Figure 6.

a) Relative viability of L929 fibroblasts in response to extract obtained from freshly prepared (24 h) and aged (7 d) SR610 samples, which contain Irgacure 2959 photoinitiator in $0.5 \%$ and $2 \%$ concentrations. b) Relative viability of L929 fibroblasts in direct contact 
with freshly prepared ( $24 \mathrm{~h}$ ) and aged (7 d) samples. For both tests, relative cell viability correlates inversely with the amount of photoinitiator. 

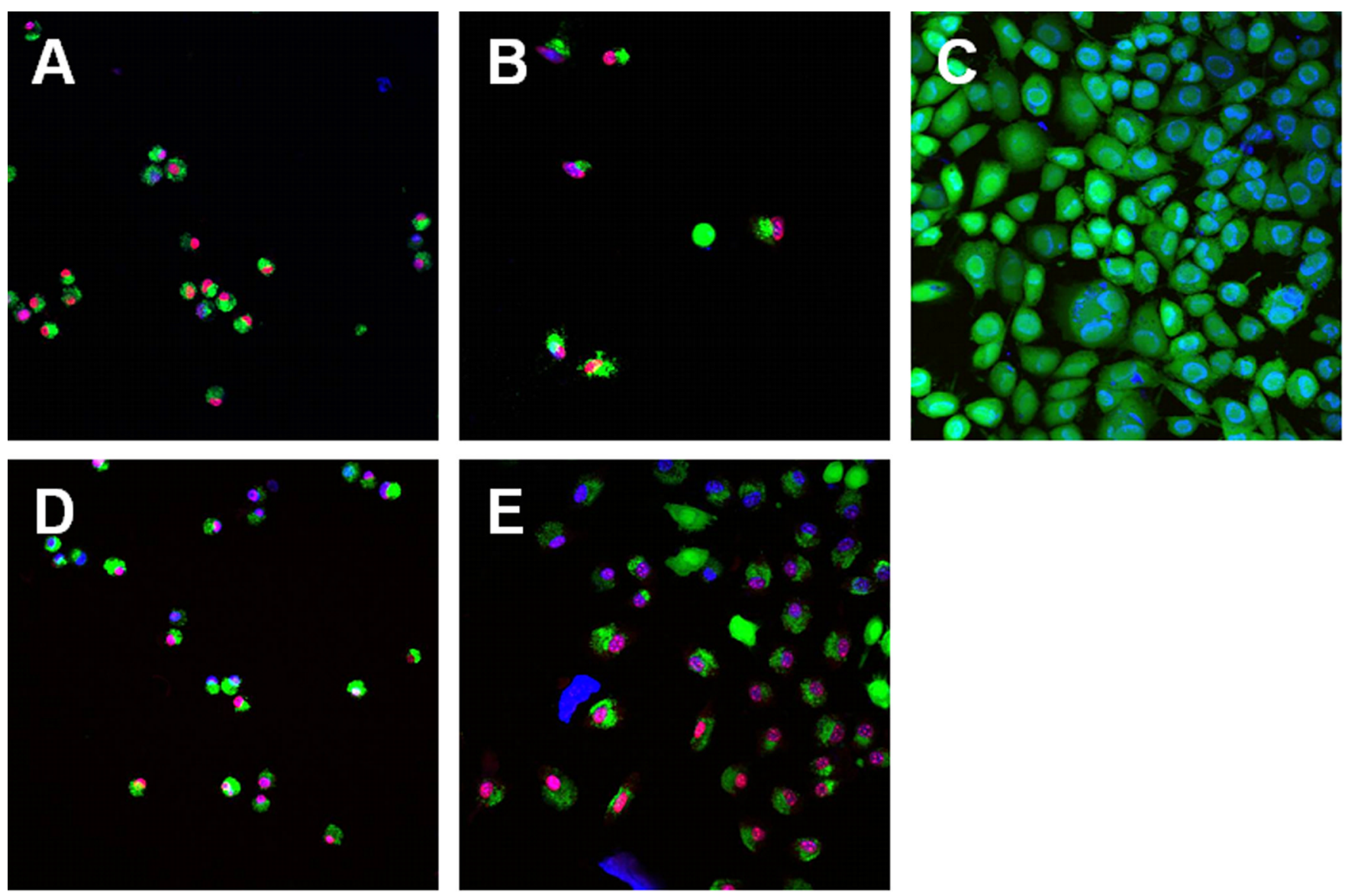

Figure 7.

Live/dead assay of cells seeded onto aged SR610 samples containing $0.5 \%$ Irgacure 2959 (A,D), $2 \%$ Irgacure 2959 (B, E), and polystyrene (C). 